\title{
Knockdown of lncRNA XIST prevents the epithelial-mesenchymal transition of TGF- $\beta 2$-induced human lens epithelial cells via miR-124/Slug axis
}

\author{
XUE JIANG ${ }^{1,2}$; HONG ZHANG ${ }^{1,2, *}$ \\ ${ }^{1}$ Eye Hospital, the First Affiliated Hospital of Harbin Medical University, Harbin, 150007, China \\ ${ }^{2}$ Key laboratory of Basic and Clinical Research of Heilongjiang Province, Harbin, 150086, China
}

Key words: LncRNA XIST, miR-124, Slug, Posterior capsular opacification, Epithelial-Mesenchymal Transition

\begin{abstract}
Posterior capsular opacification (PCO) is linked to the pathological process of lens epithelial cells, which includes proliferation, migration, and epithelial-mesenchymal transition (EMT). Our goal was to investigate whether long noncoding RNA (lncRNA) XIST contributes to EMT via targeting miR-124/Slug axis in TGF- $\beta 2$-induced SRA01/04 cells. EMT was induced by stimulating SRA01/04 cells with $10 \mathrm{ng} / \mathrm{mL}$ TGF- $\beta 2$ for $24 \mathrm{~h}$, and PCO microenvironment was simulated. The expressions levels of lncRNA XIST, miR-124, and Slug were measured by realtime polymerase chain reaction (RT-PCR) and western blot. The role and mechanism of lncRNA XIST in promoting EMT of TGF- $\beta 2$-treated SRA01/04 cells were investigated by using cell transfection, cell counting kit-8 (CCK-8), immunofluorescence staining, transwell assay, wound-healing assay, RT-PCR, western blot and dual-luciferase reporter assay. The expression of Slug and lncRNA XIST was markedly increased, while miR-124 was downregulated in TGF- $\beta 2$-treated SRA01/04 cells compared with the control group. Knockdown of lncRNA XIST reduced EMT, migration, and cell viability in TGF- $\beta 2$-induced SRA01/04 cells; moreover, it adversely modulated miR-124 and adjusted the expression of Slug in SRA01/04 cells, while these effects were diminished by co-transfection with AMOmiR-124. Our data demonstrated that IncRNA XIST functioned as a competitive endogenous RNA (ceRNA) of miR124 to modulate the expression level of Slug, thereby modulating EMT, migration, and cell viability in SRA01/04 cells. In conclusion, IncRNA XIST has a crucial role in promoting TGF- $\beta 2$-induced EMT via modulating the miR-124/Slug axis in SRA01/04 cells, and it may provide a novel therapeutic option for PCO treatment.
\end{abstract}

\section{Introduction}

Posterior capsular opacification (PCO) is one of the most common complications in recent cataract surgeries; it is also known as "secondary cataract" (Awasthi et al., 2009). Within $2-5$ years after the operation, $20 \%-40 \%$ of the patients experience vision decline due to PCO (Awasthi et al., 2009; Nibourg et al., 2015). Currently, the only effective treatment for PCO is Nd: YAG laser posterior capsulotomy, but the risk of complications still exists (Zhang and Xie, 2020). PCO mainly results from the pathological process of residual lens epithelial cells after cataract surgery and includes epithelialmesenchymal transition (EMT), proliferation, and migration. EMT is the primary causal factor for PCO development (Dong, 2019a; Zhang et al., 2017). In the process of EMT, lens

*Address correspondence to: Hong Zhang, zhanghong@hrbmu.edu.cn Received: 13 February 2021; Accepted: 19 May 2021 epithelial cells (LECs) transdifferentiate into myofibroblasts, losing cell polarity, cell-cell contact, and epithelial cell characteristics, and obtaining mesenchymal properties, such as motility, invasiveness, and antiapoptotic ability (Ashrafizadeh et al., 2020; Kim et al., 2014; Wernecke et al., 2018; Zhang et al., 2017). Therefore, inhibition of EMT can prevent the development of PCO.

Transforming growth factor- $\beta 2$ (TGF- $\beta 2$ ) is considered to be a crucial trigger for the changes associated with EMT in PCO. It plays a vital role in the activation and promotion of EMT in LECs through the Smads pathway, PI3K/Akt pathway, and other pathways. Besides, TGF- $\beta 2$ is the primary subtype of the TGF- $\beta$ family in aqueous humor (Dong, 2020; Zhang et al., 2017). Therefore, TGF- $\beta 2$ induced human lens epithelial cells (HLECs) can be used to simulate the PCO microenvironment (Wang et al., 2019; Zhang et al., 2017).

To date, a large number of noncoding RNAs (ncRNAs) have been considered to control gene expression and 
pathway activation at almost all levels, including the activation and inhibition of the EMT process (Beermann et al., 2016; Zhao et al., 2017). Among them, IncRNAs are non-protein coding transcripts that accommodate over 200 nucleotides, they are involved in cancer and other diseases, including PCO (Bach and Lee, 2018; Zhang et al., 2017; Zhang et al., 2018). One study showed that $775 \operatorname{lncRNAs}$ were anomalously expressed in HLEB-3 cells in total during TGF$\beta 2$-induced EMT compared with the control group, a total of $325 \operatorname{lncRNAs}$ were upregulated, whereas 450 were downregulated (Zhang et al., 2017). Furthermore, lncRNAs have been proven to regulate gene expression by acting as microRNA sponges or in competition with endogenous RNAs (Li et al., 2018). Nevertheless, the role of most lncRNAs in PCO has not been explored.

Recent studies have demonstrated that IncRNA $\mathrm{X}$ inactive specific transcript (XIST), is the primary regulator of $\mathrm{X}$ chromosome inactivation (XCI) - an epigenetic process that results in an equal dose of X-linked genes in female and male mammals (Loda and Heard, 2019). XIST is located on chromosome Xq13.2. LncRNA XIST is associated with the modulation of EMT in various malignant tumors (Chen et al., 2017; Shen et al., 2019). Moreover, the latest study confirmed that the expression level of lncRNA XIST was upregulated in TGF- $\beta 2$-induced primary HLECs (Dong, 2019b). However, to the best of our knowledge, the possibility that lncRNA XIST regulates the EMT process in PCO remains unclear and needs to be further explored.

The lncRNA XIST has been reported to interact with miR-124 to regulate the growth of laryngeal squamous cell carcinoma and bladder cancer (Xiao et al., 2019; Xiong et al., 2017). Decreased expression of miR-124 was found in LECs with cataracts (Wu et al., 2017a; Wu et al., 2017b). Meanwhile, miR-124 has a significant role in the modulation of EMT by focusing on Slug in regulating gastric cancer progression (Li et al., 2017). In addition, Slug is upregulated in TGF- $\beta 2$-treated HLECs and participates in the regulation of EMT in PCO (Dong et al., 2015). Consequently, we postulated that the existence of the lncRNA XIST/miR-124/Slug axis was involved in the EMT process of PCO.

No studies have explored the regulatory roles of $\operatorname{lncRNA}$ XIST and miR-124 in the EMT process of PCO. Therefore, we analyzed the regulatory relationship between lncRNA XIST, miR-124, and Slug in SRA01/04 cells induced by TGF- $\beta 2$ to identify a new potential target for the treatment of PCO.

\section{Materials and Methods}

\section{Cell culture and treatment}

SV40 T-antigen-human lens epithelial cell line SRA01/04 (Otwo Biotech, Shenzhen, China) was cultured overnight in Dulbecco's modified Eagle's medium (DMEM) supplemented with $10 \%$ fetal bovine serum (FBS) at $37^{\circ} \mathrm{C}$ and $5 \% \mathrm{CO}_{2}$ in a humidified atmosphere. SRA01/04 cells were divided into experimental groups and the control group. After the cells had reached approximately $80 \%-90 \%$ confluence, those in the experimental groups were treated by adding TGF- $\beta 2$ at different concentrations $(0,1,5,10$ $\mathrm{ng} / \mathrm{mL})$ at different times $(0,6,12,24 \mathrm{~h})$.

\section{Cell transfection}

The siRNA (siXIST) and its negative control (NC) were designed to target lncRNA XIST. MiR-124 mimics, anti-miR124 oligodeoxyribonucleotide (AMO-miR-124), and its negative control (AMO-NC) were obtained from RIBOBIO (Guangzhou, China). X-treme GENE siRNA Transfection Reagent was used in line with the manufacturer's instructions (GTS, CA, USA).

\section{$R N A$ isolation and $q R T-P C R$}

Total RNA was extracted using Trizol reagent (Invitrogen, Carlsbad, USA), and the extraction procedure complied with the instructions. The concentration of the extracted total RNA was monitored by a NanoDrop spectrophotometer (NanoDrop Technologies, Wilmington, DE). U6 was detected as the internal reference for miR-124, and GAPDH were detected as the internal reference for lncRNA XIST, and Slug. Quantitative statistics were calculated using the $2^{-\Delta \Delta \mathrm{Ct}}$ method calculation. The primer sequences used are shown in Table 1.

\section{Western blot}

Total protein was extracted from SRA01/04 cells that had been prepared for immunoblotting analysis. The membranes were capped with $5 \%$ skimmed milk for $2 \mathrm{~h}$ at room temperature and then incubated with primary antibodies at $4^{\circ} \mathrm{C}$ overnight. The following antibodies were used: GAPDH (ZSGB-BIO, China), Slug (C19G7; Cell Signaling), E-cadherin (4065; Cell Signaling), Fibronectin (FN) (ab2413, Abcam), a-SMA (ab32575, Abcam), and Vimentin (5741; Cell Signaling). The bands on the membrane were captured and quantified by the Odyssey fluorescence scanning system (LI-COR).

\section{Cell viability}

Cell counting kit-8 (CCK-8) was designed to analyze cell viability in line with the manufacturer's instructions. SRA01/04 cells were seeded into 96-well plates at a density of $1 \times 10^{4}$ cells/well and cultured for $24 \mathrm{~h}$. We added $10-\mu \mathrm{L}$ CCK-8 solution to the cells and incubated them at $37^{\circ} \mathrm{C}$. After $2 \mathrm{~h}$ of incubation, the absorbance was measured at $450 \mathrm{~nm}$ using a microplate reader (BMG LABTECH, Durham, NC, USA). Each sample was prepared in triplicate, and the experiment was repeated three times.

\section{Immunofluorescence staining}

The location and expression level of Slug in SRA01/04 cells were verified by immunofluorescence staining after treating the cells in different experimental settings, as explicitly described in the previous study (Du et al., 2020). The nuclei were stained with Hoechst dye.

\section{Luciferase reporter assay}

Online bioinformatics databases including Starbase v2.0, Targetscan, LncBase, and RNAhybrid, were exploited for target prediction. The sequences of target binding sites to be detected were cloned into pmiR-RB-REPORT ${ }^{\mathrm{TM}}$ Vectors (GenePharma, Shanghai, China), i.e., wt-XIST, mut-XIST, wt-Slug, or mut-Slug. MiR-124 mimics, AMO-miR-124, and their negative control (NC) were transfected into HEK293T cells. After treatment, a dual-luciferase reporter assay system 
(Promega, Madison, Wisconsin, USA) was utilized to detect luciferase activities. The specific experimental procedure was also described in other studies (Du et al., 2017). The related primer sequences are listed in Table 1.

\section{Transwell assay}

Cell migration capacity was monitored using Transwell platelets (Corning, New York, USA), i.e., $24 \mathrm{~h}$ after siXIST, siNC, AMO-miR-124, and AMO-NC had been transfected, SRA 01/04 cells were treated with TGF- $\beta 2$ for another $24 \mathrm{~h}$ at $37^{\circ} \mathrm{C}$. The cells with a density of $5 \times 10^{5}$ cells $/ \mathrm{mL}$ were seeded onto the upper chambers of 24-well plates and cultured in serum-free DMEM. Specific experimental details adhered to the manufacturer's instructions. The number of cells undergoing migration was measured with five random fields under an inverted microscope (Olympus Corporation, Japan).

\section{Wound-healing assay}

SRA01/04 cells were cultured into a monolayer and placed in a serum-free medium for $24 \mathrm{~h}$. After removing the medium, we scratched a straight line across the cell layer using the tip of a pipette, and then washed the cells with phosphate-buffered saline (PBS) to remove the suspended cells. siXIST, siNC, AMO-miR-124, and AMO-NC were transfected into the cells, and then the cells were induced by TGF- $\beta 2$ $(10 \mathrm{ng} / \mathrm{mL})$ for $24 \mathrm{~h}$. The data were measured according to the percentage of average distance. The scratch gap was recorded and photographed with an inverted microscope (Olympus Corporation, Japan).

\section{Statistical analysis}

Data were expressed as mean \pm standard deviation (SD) and analyzed in SPSS 16.0 software (IBM, Armonk, NY, USA) using a two-tailed Student's $t$-test and one-way Analysis of Variance (ANOVA). $p$ value $<0.05$ was classified as statistically significant.

\section{Results}

The expression levels of Slug and IncRNA XIST were increased, whereas miR-124 was decreased in TGF- $\beta 2$-induced in SRA01/04 cells

To explore whether EMT takes part in the pathogenesis of PCO and to determine the expression of various substances in the EMT cell model, we used different concentrations $(0,1,5,10$ $\mathrm{ng} / \mathrm{mL}$ ) of TGF- $\beta 2$ for treating SRA01/04 cells for $0,6,12$, and $24 \mathrm{~h}$ to establish the EMT cell model in vitro. Slug protein expression was upregulated in a concentration- and time-dependent manner with TGF- $\beta 2$ (Figs. $1 \mathrm{a}$ and 1b). According to the peak expression of Slug, the cells were treated with $10 \mathrm{ng} / \mathrm{mL}$ TGF- $\beta 2$ for $24 \mathrm{~h}$ in the follow-up experiment. Immunofluorescence results indicated that Slug protein expression increased after the cells had been induced by TGF- $\beta 2$ (Fig. 1c). The expression of Slug mRNA and lncRNA XIST increased, while the expression of miR-124 decreased when treated with $10 \mathrm{ng} / \mathrm{mL}$ TGF- $\beta 2$ for $24 \mathrm{~h}$ (Figs. 1d-1f). We used western blot to examine the expression of EMT-related markers, including epithelial markers (E-cadherin), and mesenchymal markers (FN, a-SMA, and vimentin). The results reflected that the protein

TABLE 1

Primers for expressing vectors, luciferase reporter plasmids and miR-124 quantification

\begin{tabular}{ll}
\hline Name & Sequence (5'-3') \\
\hline Slug-F & ATAGAATTCCCAGACCCGCTGGCAAGATG \\
Slug-R & AATGGATCCCGAGTAAACATTGATTGCGTCA \\
Slug-UTR-F & ATACTCGAGATGACAAATAAAGTCCAAAGG \\
Slug-UTR-R & ATACTCGAGATGACAAATAAAGTCCAAAGG \\
Slug-UTR-Mut-F & GCCAGACGCGAACTCAGCTCCGTAAAAAAGTATTCCAAGT \\
Slug-UTR-Mut-R & ACTTGGAATACTTTTTTACGGAGCTGAGTTCGCGTCTGGC \\
miR-124-RT & GTCGTATCCAGTGCAGGGTCCGAGGTATTCGCACTGGATACGACGGCATTCT \\
miR-124-F & GATACTCATAAGGCACGCGG \\
miR-124-R & GTGCAGGGTCCGAGGT \\
U6-F & CGCTTCGGCAGCACATATAC \\
U6-R & CAGGGGCCATGCTAATCTT \\
XIST-F & AGCTCCTCGGACAGCTGTAA \\
XIST-R & CTCCAGATAGCTGGCAACC \\
XIST siRNA-1-F & AUACUUGGGCCUUCUAUCCATT \\
XIST siRNA-1-R & GAUAGAAGGCCCAAA GUAUAATT \\
XIST siRNA-2-F & UUUUGAAGCAUAUUUUGGCUUTT \\
XIST siRNA-2-R & GCCAAAAUAUGCUUCAAAAGATT \\
XIST siRNA-3-F & UAAUGAUGCAUAUUUUGGCUUTT \\
XIST siRNA-3-R & GCUAGAAUGUCGUUCAAAGGATT \\
GAPDH-F & AGAAGGCTGGGGCTCATTTG \\
GAPDH-R & AGGGGCCATCCACAGTCTTC \\
\hline
\end{tabular}




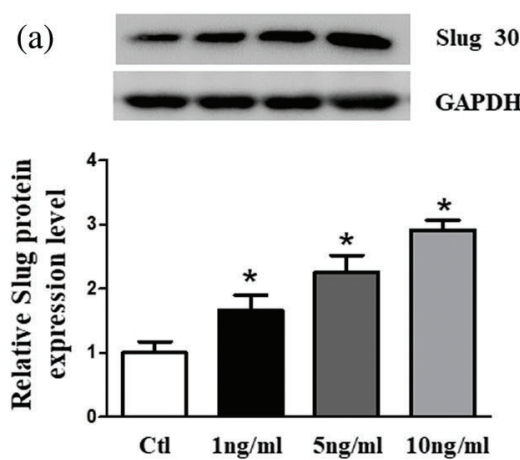

(b)
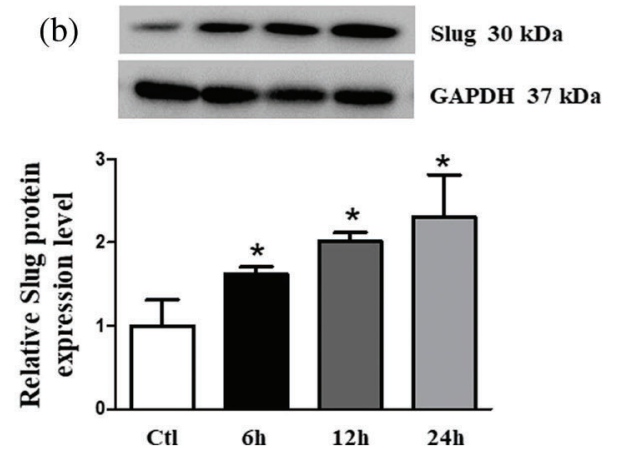

(c)

Ctl

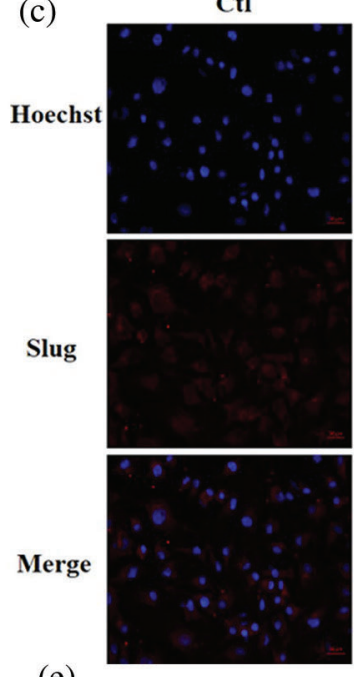

(e)

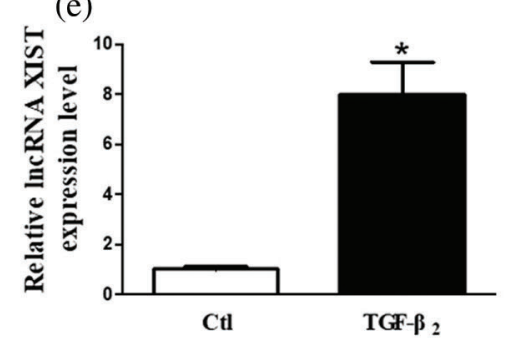

(g)

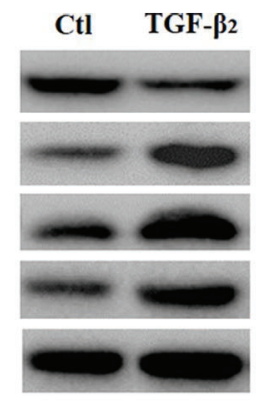

E-cadherin 120 kDa

Fibronectin(FN) $285 \mathrm{kDa}$

a-SMA 42 kDa

Vimentin 57 kDa

GAPDH 37 kDa
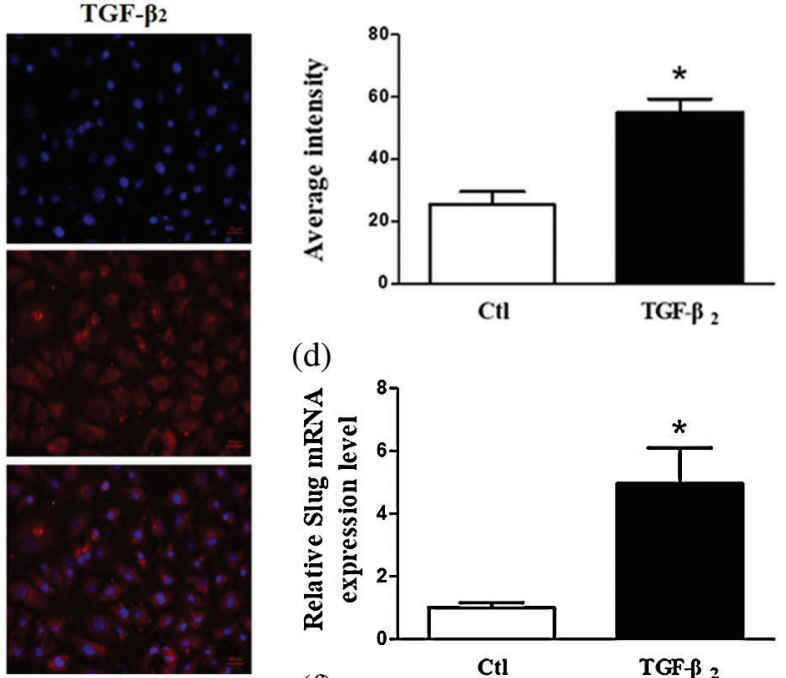

(d)

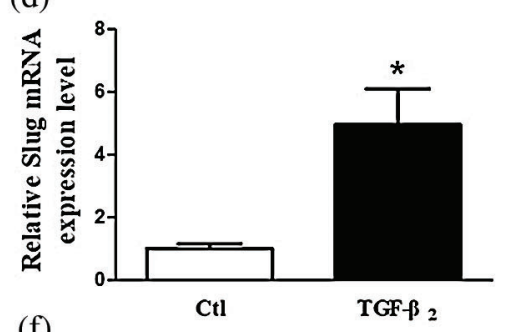

(f)
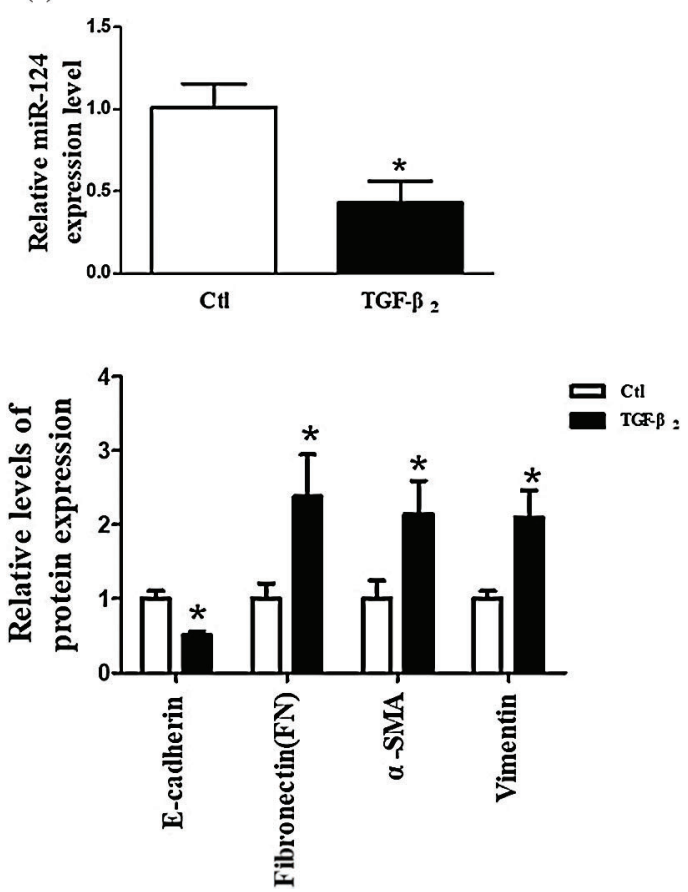

FIGURE 1. The expression levels of Slug and IncRNA XIST increased, whereas miR124 decreased after treatment of SRA01/04 cells with TGFß2. (a) SRA01/04 cells were induced with different concentrations $(0,1,5,10 \mathrm{ng} / \mathrm{mL})$ of TGF- $\beta 2$ for $24 \mathrm{~h}$. (b) TGF$\beta 2(10 \mathrm{ng} / \mathrm{mL})$ was used to induce SRA01/04 cells for 0, 6, 12, and 24 h. (c) The average intensity of Slug protein expression increased in SRA01/04 cells induced by TGF- $\beta 2$ compared with the control (Ctl) group. (d) The expression of Slug mRNA in SRA01/04 cells induced by $10 \mathrm{ng} / \mathrm{mL}$ TGF- $\beta 2$ for $24 \mathrm{~h}$. (e) The expression of lncRNA XIST in SRA01/04 cells induced by $10 \mathrm{ng} / \mathrm{mL}$ TGF- $\beta 2$ for $24 \mathrm{~h}$. (f) The expression of miR-124 in SRA01/04 cells induced by $10 \mathrm{ng} / \mathrm{ml}$ TGF- $\beta 2$ for $24 \mathrm{~h}$. (g) EMT markers in SRA01/04 cells induced by TGF- $\beta 2 .{ }^{*} p<0.05$ vs. Ctl group. Data are expressed as mean \pm SD. $\mathrm{N}=3$ batches of cells for each group. expression of E-cadherin decreased, while the protein levels of FN, $\alpha$-SMA, and vimentin increased in SRA01/04 cells (Fig. 1g).

Knockdown of IncRNA XIST had a reverse effect on TGF- $\beta 2$ induced EMT, cell viability, and migration in SRA01/04 cells To further investigate the biological function of lncRNA XIST, we transfected XIST siRNA (siXIST) and screened for the best knockdown efficiency to alleviate endogenous lncRNA XIST in SRA01/04 cells (Fig. 2a). The role of lncRNA XIST on cell viability was evaluated using CCK-8 assay, which indicated that inhibition of lncRNA XIST effectively suppressed the increased cell viability induced by TGF- $\beta 2$ stimulation of SRA01/04 cells (Fig. 2b). Additionally, the wound healing assay and transwell assay revealed that the knockdown of lncRNA XIST remarkably inhibited the SRA01/04 cell migration induced by TGF- $\beta 2$ (Figs. $2 c$ and $2 d$ ). 

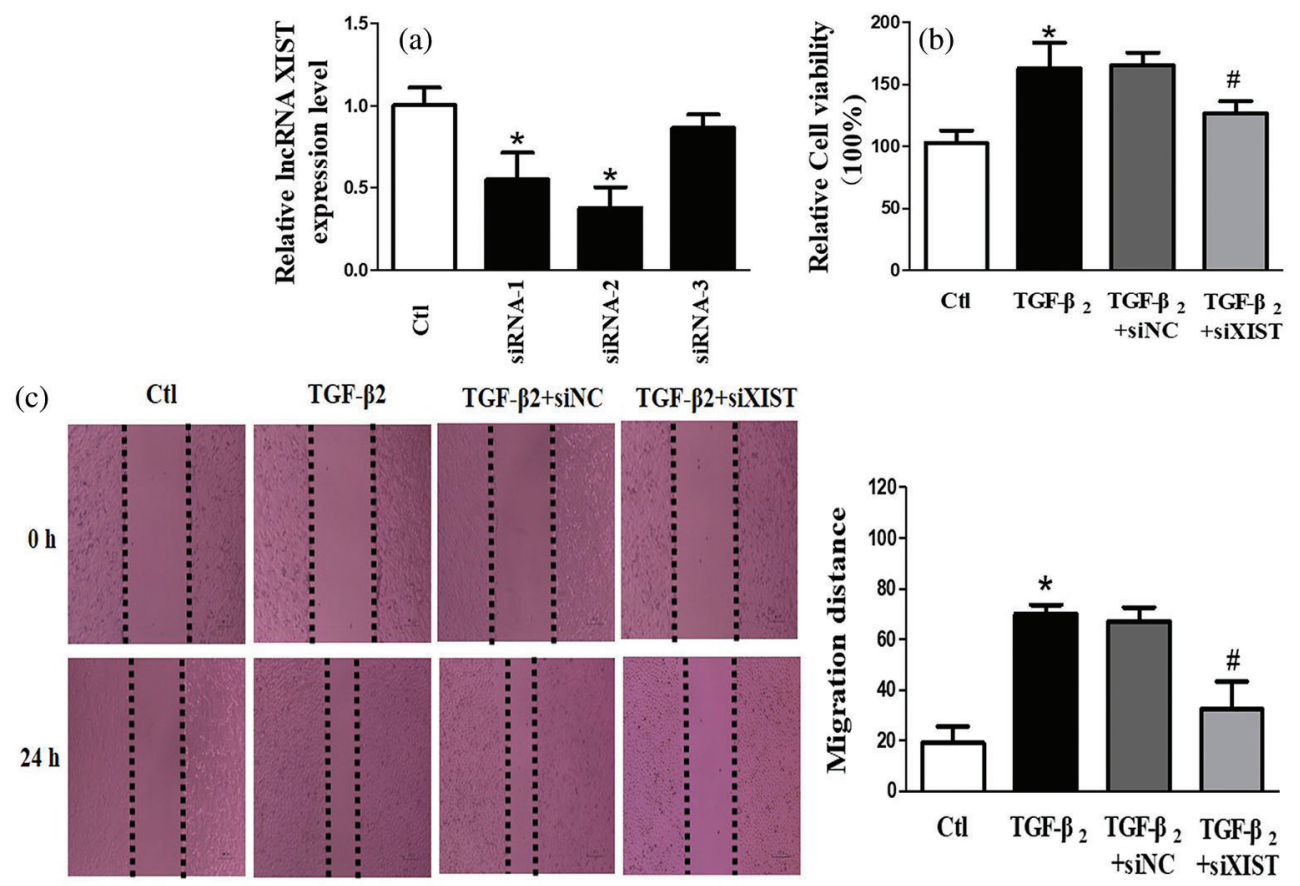

(d)

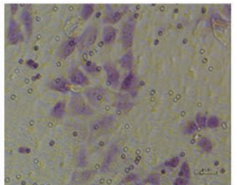

Ctl

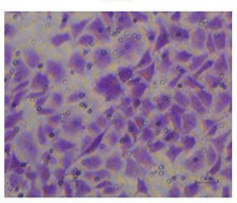

TGF- $\boldsymbol{\beta}_{2}+$ siNC

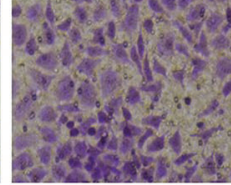

TGF- $\beta 2$

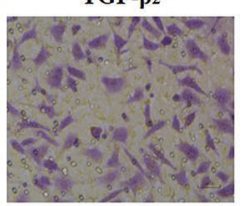

TGF- $\boldsymbol{\beta}_{2}+$ siXIST
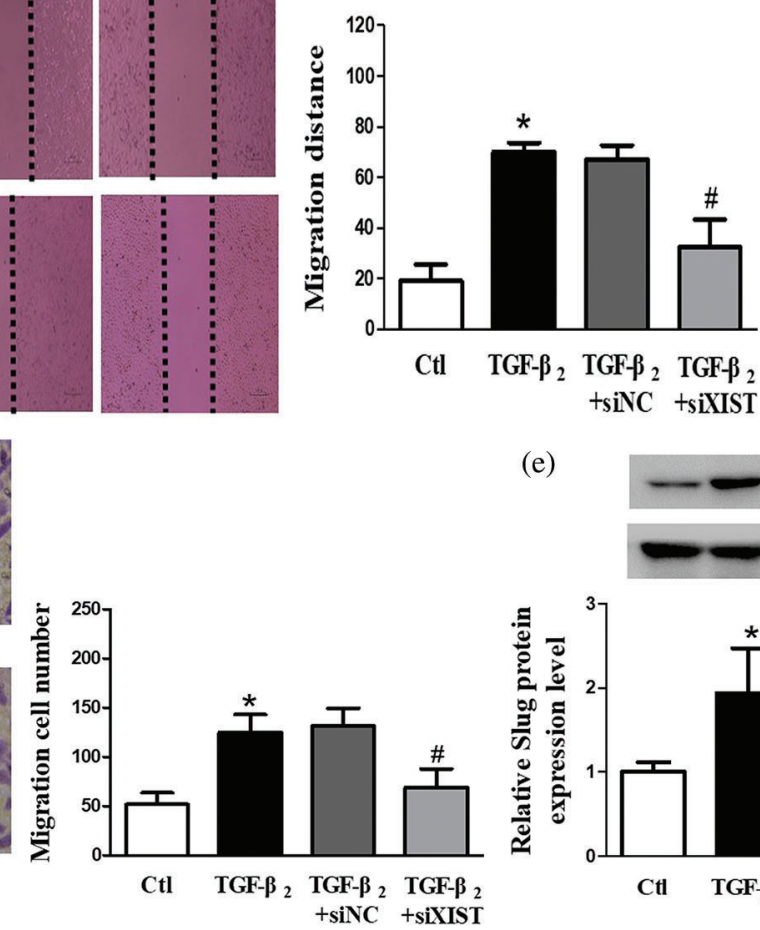

(e)
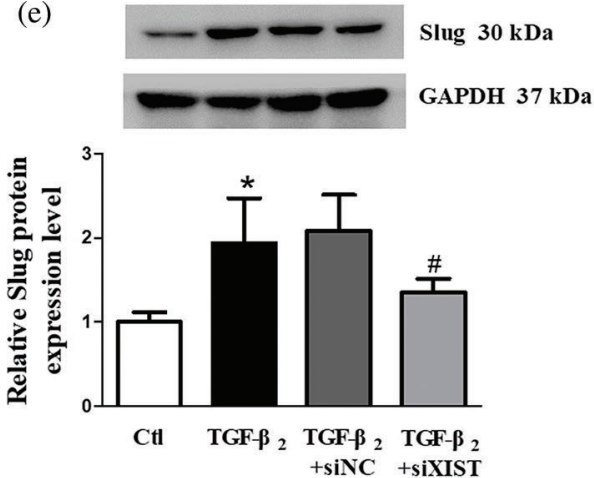

(f)

E-cadherin $120 \mathrm{kDa}$

Fibronectin(FN) 285 kDa

a-SMA 42 kDa

Vimentin 57 kDa

GAPDH 37 kDa

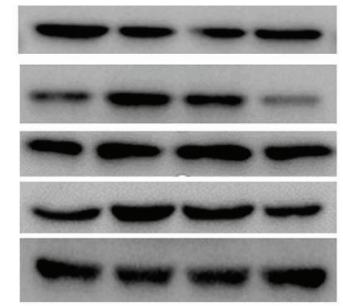

$\begin{array}{rllll}\text { Ctl } & + & - & - & - \\ \text { TGF- } \beta 2 & - & + & + & + \\ \operatorname{siNC} & - & - & - & + \\ \operatorname{siXIST} & - & - & + & -\end{array}$
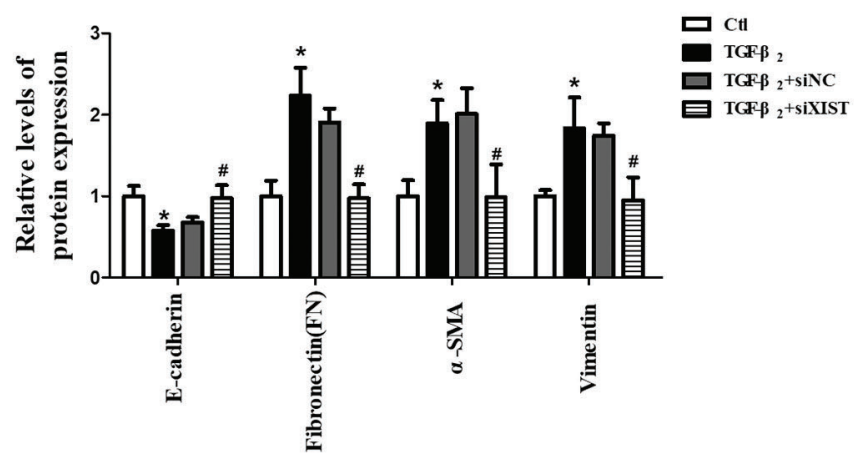

FIGURE 2. Knockdown of lncRNA XIST had a reverse effect on TGF- $\beta 2$ induced EMT, cell viability, and migration in SRA01/04 cells. (a) We constructed the cell transfection model by silencing lncRNA XIST and verified the effectiveness of transfection. (b) CCK-8 assay was used to detect cell viability. (c) Wound-healing assay was used to test cell migration. (d) Transwell assay was used to test cell migration. (e) The expression of Slug protein in the TGF- $\beta 2$ group was significantly increased compared with control (Ctl) group, while Slug protein expression in the TGF- $\beta 2+$ siXIST group was considerably lower than that in the TGF- $\beta 2+$ siNC group. (f) Western blot was used to detect the expression of EMT-related markers (E-cadherin, FN, $\alpha$-SMA, and vimentin). ${ }^{*} p<0.05 v$ s. Ctl group. ${ }^{*} p<0.05 v s$. TGF- $\beta 2+$ siNC group. Data are expressed as mean $\pm \mathrm{SD}$. $\mathrm{N}=3$ batches of cells for each group.

As shown in Fig. 2e, suppressed lncRNA XIST reversed the elevated protein expression of Slug induced by TGF- $\beta 2$. E-cadherin expression was obviously downregulated, while the expression levels of $\mathrm{FN}, \alpha$-SMA and vimentin were significantly upregulated in TGF- $\beta 2$-treated SRA01/04 cell line. Still, the interference of IncRNA XIST could reverse these effects on EMT-related markers (Fig. 2f). These results suggested that lncRNA XIST had a regulatory role in TGF- $\beta 2$ induced EMT, cell viability, and migration in SRA01/04 cells.

Target prediction between IncRNA XIST and miR-124, as well as between miR-124 and Slug

According to the results presented above, we noticed that lncRNA XIST expression highly correlated with Slug. 
Previous studies showed that MiR-124 was able to target lncRNA XIST and Slug. Therefore, we speculated that there was a potential regulatory relationship between lncRNA XIST, miR-124, and Slug. We used biological prediction tools to examine the potential binding sites between lncRNA XIST and miR-124, as well as between miR-124 and Slug (Fig. 3a). The relative luciferase activities significantly decreased when miR-124 mimics and wt-XIST or wt-Slug-3'UTR were cotransfected into the cells. Conversely, the relative luciferase activities increased when AMO-miR-124 and wt-XIST or wtSlug-3'UTR were co-transfected into the cells. No significant changes were found in fluorescence activity at the mutant binding sites of XIST or Slug-3'UTR (Figs. 3b and 3c). To further explore the regulatory role of miR-124 on Slug expression, with miR-124 mimics and AMO-miR-124 transfected, Slug expression was detected by western blot. As shown in Figs. $3 d-3 g$, overexpressed miR-124 decreased the expression of lncRNA XIST as well as Slug mRNA and protein. Moreover, the expression levels of lncRNA XIST and Slug mRNA and protein were upregulated with AMO-miR124 transfection. As shown in Figs. $3 \mathrm{~h}$ and $3 \mathrm{i}$, silencing IncRNA XIST inhibited Slug expression, while miR-124 mimics was able to further inhibit Slug expression. AMOmiR-124 reversed the decrease of Slug expression caused by the knockdown of lncRNA XIST. Collectively, the results demonstrated that IncRNA XIST acted in a competing endogenous RNA (ceRNA) manner by targeting miR-124 to affect miR-124 expression, thereby regulating Slug expression.

AMO-miR-124 had a reversed effect of silencing $\operatorname{lncRNA}$ XIST on the expression of Slug, EMT, cell viability and migration induced by TGF- $\beta 2$

To verify that the influence of IncRNA XIST on the regulation of Slug was mediated by miR-124, SRA01/04 cells were (a)

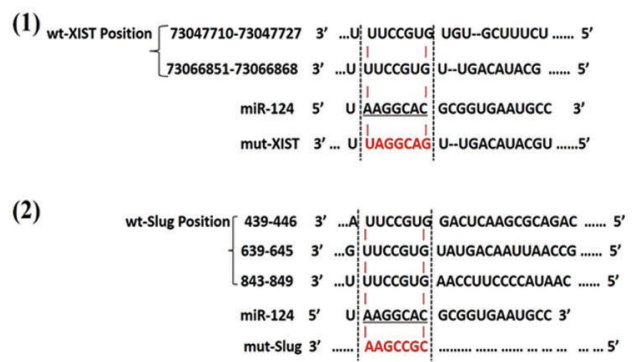

(d)

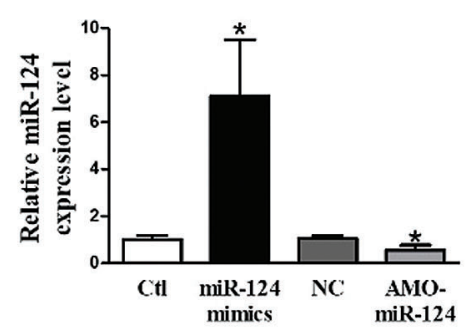

(g)

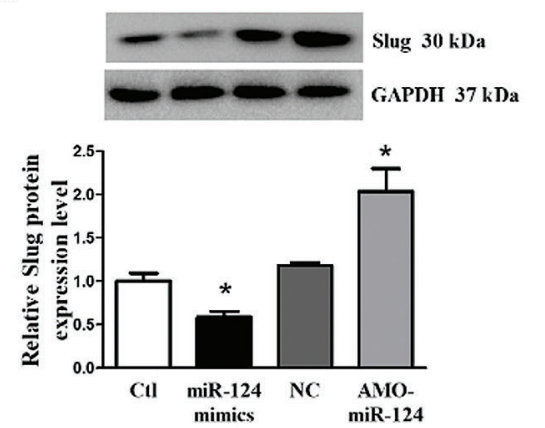

(b)

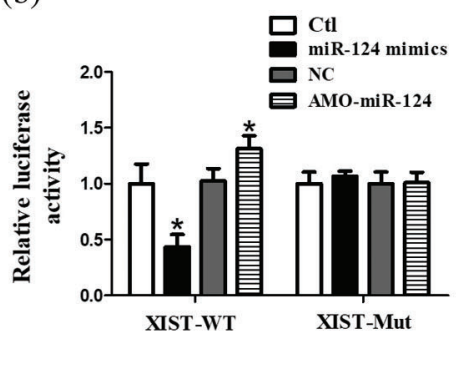

(e)

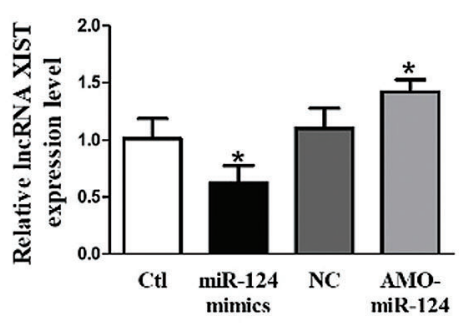

(h)

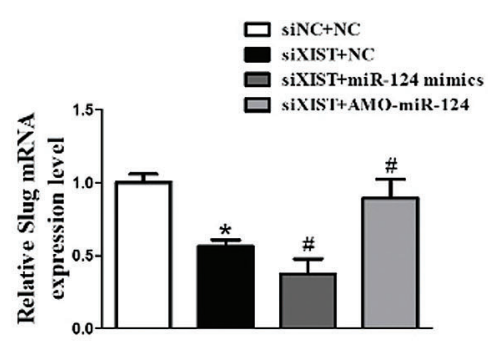

(c)
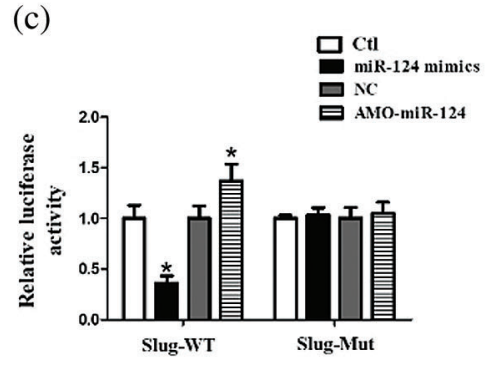

(f)

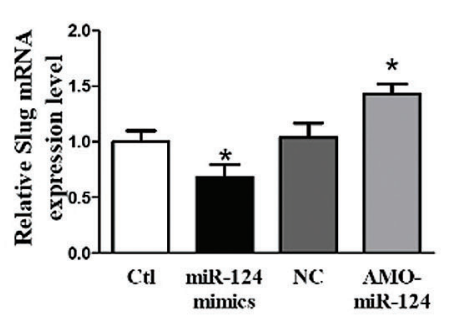

(i)

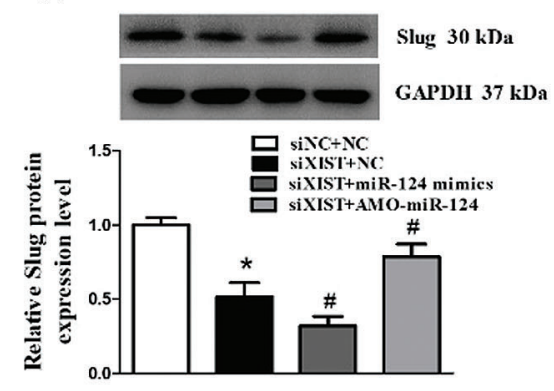

FIGURE 3. Target prediction between lncRNA XIST and miR-124. Slug directly targeted miR-124. (a) Target prediction between lncRNA XIST and miR-124, as well as between miR-124 and Slug, by bioinformatic analysis. (a-1) The bioinformatic analysis demonstrated that two predicted binding sites of wt-XIST (wild type) directly targeted with miR-124. Mut-XIST (mutant) contained mutations in the binding site within the XIST sequence. (a-2) Bioinformatic analysis indicated that miR-124 directly targeted three predicted binding sites of 3'UTR of the Slug gene (wild type). Mut-Slug (mutant) contained mutations in the binding site within the Slug sequence. (b) LncRNA XIST targeted with miR-124 directly by dual-luciferase reporter assay. (c) Slug targeted miR-124 directly by dual-luciferase reporter assay. (d) The expression level of miR-124 transfected with miR-124 mimics or AMO-miR-124. (e) The expression level of lncRNA XIST transfected with miR-124 mimics or AMO-miR-124. (f) The expression level of Slug mRNA transfected with miR-124 mimics or AMO-miR-124. (g) The expression level of Slug protein transfected with miR-124 mimics or AMO-miR-124. (h) The expression level of Slug mRNA with co-transfection of siXIST and miR-124 mimics, or siXIST and AMO-miR-124. (i) The expression level of Slug protein with co-transfection of siXIST and miR-124 mimics, or siXIST and AMO-miR-124. ${ }^{*} p<0.05$ vs. NC group. ${ }^{*} p<0.05 v$ s. siXIST + NC group. Data are expressed as mean \pm SD. $\mathrm{N}=3$ batches of cells for each group. 
transfected with siXIST accompanied by TGF- $\beta 2$ stimulation. The expression level of miR-124 decreased, while Slug increased under TGF- $\beta 2$ treatment of SRA 01/04 cells. However, the suppression of lncRNA XIST alleviated the effect of TGF- $\beta 2$ on miR-124 and Slug, these effects were reversed with AMO-miR-124 co-transfection (Figs. 4a-4c). Besides, AMO-miR-124 inversed the role of suppressing lncRNA XIST on cell viability, migration, and EMT in
TGF- $\beta 2$-treated SRA01/04 cells (Figs. $4 \mathrm{~d}-4 \mathrm{~g}$ ). These findings demonstrated that the knockdown of lncRNA XIST diminished the TGF- $\beta 2$-induced influence on EMT via miR124/Slug axis in SRA01/04 cells, and AMO-miR-124 was able to reverse the changes in EMT-related proteins induced by silencing lncRNA XIST. Therefore, the lncRNA XIST/miR$124 /$ Slug axis participated in the progression of EMT in TGF$\beta 2$-treated SRA01/04 cells.

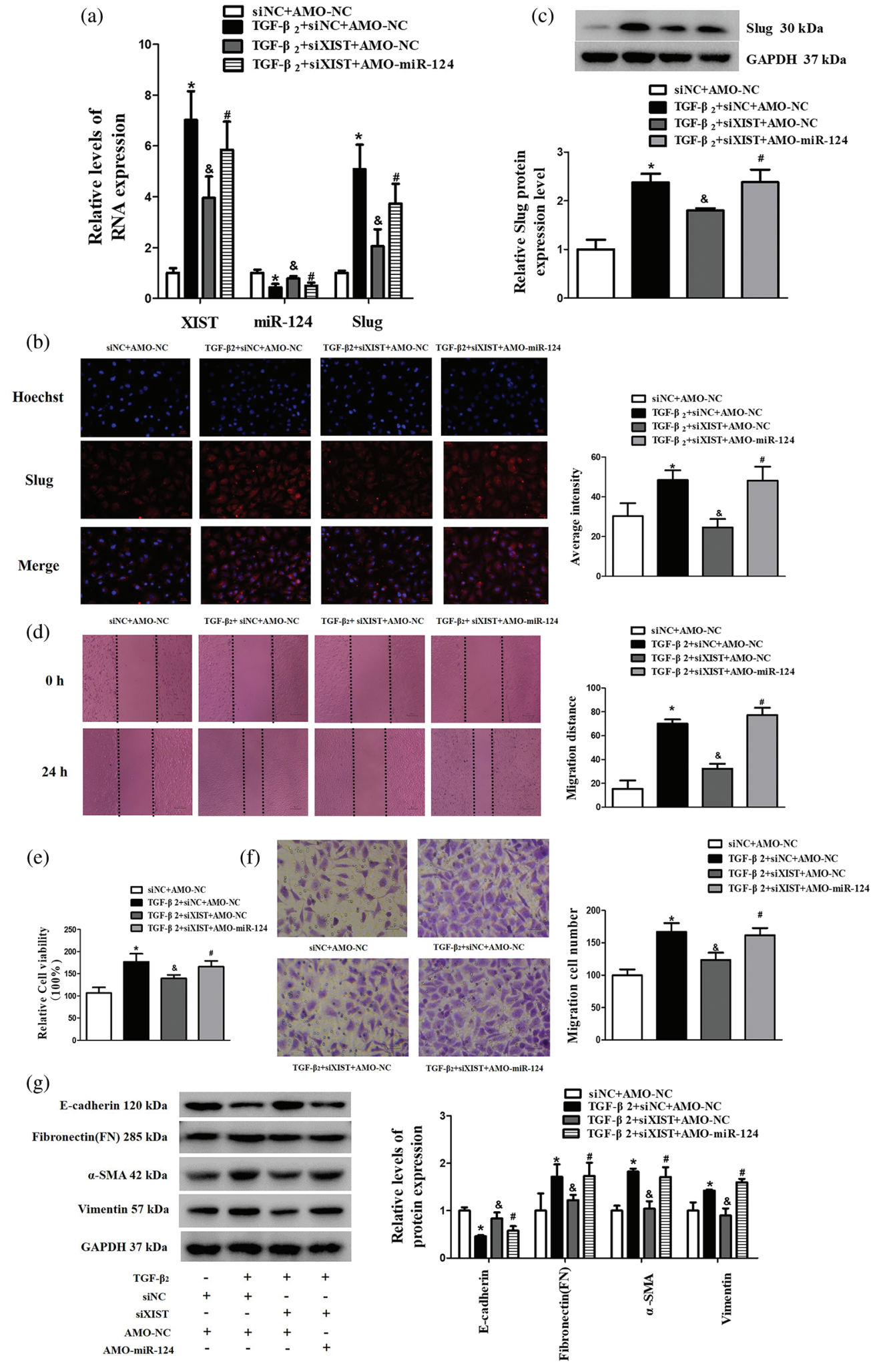

FIGURE 4. AMO-miR-124 reversed the effect of silencing lncRNA XIST on the expression of Slug, EMT, cell viability, and migration induced by TGF- $\beta 2$. (a) The RNA expression level of lncRNA XIST, miR-124, and Slug transfected with siXIST and AMO-miR-124. (b) The average intensity of Slug protein expression transfected with siXIST and AMO-miR-124 was detected by immunofluorescence. (c) The expression of Slug protein transfected with siXIST and AMO-miR-124 was detected by western blot. (d) Wound-healing assay was used to detect the migration distance in SRA01/04 cells tansfected with siXIST and AMO-miR-124. (e) CCK-8 assay was used to detect the cell viability in SRA01/04 cells transfected with siXIST and AMOmiR-124. (f) Transwell assay was used to detect the migration cell number in SRA01/04 cells transfected with siXIST and AMO-miR-124. (g) Western blot was used to detect the expression of EMTassociated markers in SRA01/04 cells transfected with siXIST and AMO-miR-124. ${ }^{\star} p<0.05$ vs. siNC+AMO-NC group. ${ }^{\circledR} p<0.05$ vs. TGF- $\beta 2+$ siNC+AMO-NC group. ${ }^{\#} p<0.05$ vs. TGF- $\beta 2+$ siXIST + AMO-NC group. Data are expressed as mean $\pm \mathrm{SD}$. $\mathrm{N}=3$ batches of cells for each group. 


\section{Discussion}

PCO is the most common complication after cataract surgery. It mainly develops due to cell viability, migration, and EMT of residual HLECs postoperatively (Li et al., 2019) ; specifically, EMT likely plays a core role in the PCO formation (Dong, 2019a), but the underlying mechanisms of molecular regulation in the development of PCO remain unclear. In the current study, we showed that the expression of EMTassociated markers, cell migration, and cell viability in SRA01/04 cells induced by TGF- $\beta 2$ were modulated through a lncRNA XIST-dependent mechanism. Specifically, lncRNA XIST negatively regulated miR-124, and miR-124 targeted Slug (snail 2) directly. Finally, we proved that the lncRNA XIST/miR-124/Slug axis participated in the EMT of HLECs induced by TGF- $\beta 2$.

LncRNA XIST is the first lncRNA identified on chromosome Xq13.2. It is dysregulated in a wide variety of human cancers, and it plays an integral role in the development and progression of tumors by regulating cell viability, invasion, migration, and EMT(Hai et al., 2020; Hu et al., 2018; Shen et al., 2019; Wang et al., 2018; Xiao et al., 2019). For example, elevated lncRNA XIST was reportedly found in pancreatic cancer cells and tissues, and the knockdown of lncRNA XIST in pancreatic cancer cell lines inhibited EMT by suppressing mesenchymal markers $(\beta$ catenin and Snail) and upregulating epithelial markers (Ecadherin and Claudin-1) (Shen et al., 2019). Another study showed that elevated lncRNA XIST expression was closely associated with poor overall survival in colorectal cancer patients and promoted metastasis and EMT in colorectal cancer (Chen et al., 2017). LncRNA XIST was upregulated in TGF- $\beta 2$-treated HLECs (Dong, 2019b), which is in agreement with our findings. Therefore, we demonstrated that silencing lncRNA XIST can inhibit EMT in TGF- 32 induced SRA01/04 cells.

LncRNA XIST acts as a sponge and can represent biological characteristics via a ceRNA mechanism (Hai et al., 2020). Several previous findings indicated that lncRNA XIST could adversely modulate miR-124 via sponging miR124 , thus regulating various cancers, including laryngeal squamous cell carcinoma and bladder cancer (Xiao et al., 2019; Xiong et al., 2017). The action of miR-124 in PCO has not been investigated, except that some previous studies showed that miR-124 expression was significantly decreased in LECs of cataract (Wei and Sun, 2019; Wu et al., 2017a; Wu et al., 2017b). In the current study, we demonstrated that the expression level of miR-124 decreased in TGF- $\beta 2$ induced SRA01/04 cells. Interestingly, by luciferase reporter assay, we also found that lncRNA XIST was able to adversely modulate miR-124 via functioning as a ceRNA of miR-124, which is consistent with the previous report (Du et al., 2020).

In addition to the TGF- $\beta$ signaling pathway, the activation of crucial EMT transcriptional repressors, such as Snai1, Slug, Twist1, Twist2, ZEB1, and ZEB2, could suppress $\mathrm{CDH} 1$ (E-cadherin promoter) in a variety of different routes in cancers (Derynck et al., 2021; Puisieux et al., 2014). CDH1 expression deficiency has been recognized as a characteristic feature of EMT (Liang et al., 2018). However, the underlying molecular events that drive the EMT process during the progression of PCO are still largely unknown. Slug is a biomarker of EMT and has been reported to be upregulated in PCO-attached LECs and HLECs induced by TGF- $\beta$ (Chandler et al., 2007; Dong et al., 2015; Wang et al., 2016; Zhang et al., 2018), which is in agreement with our results. Meanwhile, E-cadherin expression was downregulated, while the expression levels of FN, a-SMA, and vimentin were increased, which is in agreement with the former findings (Dong et al., 2014; Zhang et al., 2018). The data showed the EMT cell model was successfully established. We confirmed that Slug was participated in the etiopathogenesis of PCO. Besides, we found binding sites between miR-124 and Slug by bioinformatic prediction, consistent with previous reports (Cui and $\mathrm{Hu}, 2016$ ) .

We further demonstrated that the knockdown of lncRNA XIST can upregulate miR-124 expression and downregulate the expression of Slug protein in TGF- $\beta 2$ treated SRA01/04 cells. Our findings further indicated that co-transfection of AMO-miR-124 can reverse the downregulation of Slug induced by siXIST, which is consistent with the result that miR-124 regulates Slug expression (Cui and Hu, 2016; Liang et al., 2013). In addition, siXIST attenuated the impact of TGF- $\beta 2$ on the expression of miR-124 and Slug and the process of cell viability, migration, and EMT, but these effects were reversed by co-transfection of AMO-miR-124. These findings suggested that IncRNA XIST regulated TGF- $\beta 2$ induced Slug protein expression by competitively interacting with miR-124. Overall, these results indicated that the knockdown of lncRNA XIST can protect HLECs treated by TGF- $\beta 2$ from EMT progression via regulating the miR-124/Slug pathway.

The limitation of this study is that although the TGF- $\beta 2$ induced SRA01/04 cell line is a common in vitro cell model for PCO research (Wang et al., 2019; Zhang et al., 2018), it may not reflect normal lens biology because it does not express many normal lens markers; thus, we cannot determine whether there are potential regulatory differences when compared with the normal lens (Weatherbee et al., 2019). Besides, we only verified the regulatory axis at the cellular level, but not in the animal model. We will do further research in the follow-up experiments. Another interesting point that needs to be discussed is that this study's purpose was to reveal a downstream regulatory signaling pathway of lncRNA XIST in the pathological development of PCO, which is the common mechanism of PCO in female and male individuals. LncRNA XIST is an X chromosome-related lncRNA. Its differential expression is tightly associated with the gender-related incidence of diseases, such as breast cancer and cervical cancer (Chen et al., 2019; Zong et al., 2020). Still, lncRNA XIST is also involved in some non-gender-related diseases, such as diabetic retinopathy (Dong et al., 2020). One research study showed that the sex ratio of diabetic retinopathy was not uniform. It was reported that the incidence rate in men and women was similar in the study of the Nakuru (Bastawrous et al., 2017; Sabanayagam et al., 2019). Several epidemiologic studies have suggested that the incidence of PCO is higher in elderly women than in agematched men (Congdon et al., 2008; Fong et al., 2014; 
Lee et al., 2016). Nevertheless, this is not a uniform conclusion, and other studies do not support this view (Westling and Calissendorff, 1991). Therefore, the existence of gender differences in PCO should be studied. In addition, there are many factors affecting PCO, such as intraocular lens material and design, surgical techniques, and age (Awasthi et al., 2009; Nibourg et al., 2015). We will further explore the activation mechanism of lncRNA XIST upstream in X chromosome based on this pathway to indirectly explain whether there is a gender difference trend in PCO, which will have important clinical significance.

In conclusion, the current study revealed that the knockdown of lncRNA XIST alleviated EMT-associated Slug protein alterations by sponging miR-124. We demonstrated the existence of the lncRNA XIST/miR-124/Slug axis in the TGF- $\beta 2$-induced SRA01/04 cell line. TGF- $\beta 2$-induced SRA01/04 cell line is a common cell model to study EMT progression in PCO. Therefore, we speculated that the lncRNA $\mathrm{XIST} / \mathrm{miR}-124 /$ Slug axis may be related to the pathogenesis of PCO. Together, the results disclosed novel lncRNA and miRNA participating in the EMT cell model of PCO, suggesting that the IncRNA XIST/miR-124/Slug axis has the potential to be a promising target for clinical treatment of PCO.

Availability of Data and Materials: All data generated or analysed during this study are included in this article.

Authors' Contributions: Xue Jiang conceived and designed the experiments. Xue Jiang carried out the experiments and contributed to the writing of the manuscript. Xue Jiang and Hong Zhang participated in the design and implementation of the project, raised funds, and completely revised the manuscript. All authors reviewed the results and approved the final version of the manuscript.

Funding Statement: This work was supported by the Joint Foundation for Regional Innovation and Development (U20A20363), the Natural Science Foundation of Heilongjiang Province (LH2020H039), and the National Natural Science Foundation of China (No. 81970776).

Conflicts of Interest: The authors declare that they have no conflicts of interest to report regarding the present study.

\section{References}

Ashrafizadeh M, Hushmandi K, Hashemi M, Akbari ME, Kubatka P et al. (2020). Role of microRNA/epithelial-to-mesenchymal transition axis in the metastasis of bladder cancer. Biomolecules 10: 1159.

Awasthi N, Guo S, Wagner BJ (2009). Posterior capsular opacification: A problem reduced but not yet eradicated. Archives of Ophthalmology 127: 555-562.

Bach DH, Lee SK (2018). Long noncoding RNAs in cancer cells. Cancer Letters 419: 152-166.

Bastawrous A, Mathenge W, Wing K, Bastawrous M, Rono $\mathrm{H}$ et al. (2017). The incidence of diabetes mellitus and diabetic retinopathy in a population-based cohort study of people age 50 years and over in Nakuru. Kenya BMC Endocrine Disorders 17: 19.

Beermann J, Piccoli MT, Viereck J, Thum T (2016). Non-coding RNAs in development and disease: Background, mechanisms, and therapeutic approaches. Physiological Reviews 96: 1297-1325.
Cui Z, Hu Y (2016). MicroRNA-124 suppresses Slug-mediated lung cancer metastasis. European Review for Medical and Pharmacological Sciences 20: 3802-3811.

Chandler HL, Barden CA, Lu P, Kusewitt DF, Colitz CM (2007). Prevention of posterior capsular opacification through cyclooxygenase-2 inhibition. Molecular Vision 13: 677-691.

Chen DL, Chen LZ, Lu YX, Zhang DS, Zeng ZL et al. (2017). Long noncoding RNA XIST expedites metastasis and modulates epithelial-mesenchymal transition in colorectal cancer. Cell Death \& Disease 8: e3011.

Chen X, Xiong D, Ye L, Wang K, Huang L et al. (2019). Up-regulated lncRNA XIST contributes to progression of cervical cancer via regulating miR-140-5p and ORC1. Cancer Cell International 19: 45.

Congdon N, Fan H, Choi K, Huang W, Zhang L, Zhang S, Liu K, Hu IC, Zheng Z, Lam DS (2008). Impact of posterior subcapsular opacification on vision and visual function among subjects undergoing cataract surgery in rural China: Study of Cataract Outcomes and Up-Take of Services (SCOUTS) in the Caring is Hip Project, report 5. The British Journal of Ophthalmology 92: 598-603.

Derynck R, Turley SJ, Akhurst RJ (2021). TGFbeta biology in cancer progression and immunotherapy. Nature Reviews Clinical Oncology 18: 9-34.

Dong N (2019a). Long noncoding RNA MALAT1 acts as a competing endogenous RNA to regulate TGF- $\beta 2$ induced epithelial-mesenchymal transition of lens epithelial cells by a microRNA-26a-dependent mechanism. Biomed Research International 2019: 1569638.

Dong N (2019b). Long noncoding RNA MALAT1 acts as a competing endogenous RNA to regulate TGF- $\beta 2$ induced epithelial-mesenchymal transition of lens epithelial cells by a MicroRNA-26a-dependent mechanism. Biomed Research International 2019: 1569638.

Dong N (2020). Long noncoding RNA NEAT1 regulates TGF- $\beta 2-$ induced epithelial-mesenchymal transition of lens epithelial cells through the miR-34a/Snaill and miR-204/Zeb1 pathways. Biomed Research International 2020: 8352579.

Dong N, Tang X, Xu B (2015). miRNA-181a inhibits the proliferation, migration, and epithelial-mesenchymal transition of lens epithelial cells. Investigative Ophthalmology \& Visual Science 56: 993-1001.

Dong N, Xu B, Benya SR, Tang X (2014). MiRNA-26b inhibits the proliferation, migration, and epithelial-mesenchymal transition of lens epithelial cells. Molecular and Cellular Biochemistry 396: 229-238.

Dong Y, Wan G, Peng G, Yan P, Qian C, Li F (2020). Long noncoding RNA XIST regulates hyperglycemia-associated apoptosis and migration in human retinal pigment epithelial cells. Biomedicine \& Pharmacotherapy 125: 109959.

Du Y, Gao GQ, Li JX, Suo S, Liu S et al. (2020). Silencing of long noncoding RNA XIST attenuated Alzheimer's diseaserelated BACE1 alteration through miR-124. Cell Biology International 44: 630-636.

Du Y, Zhang M, Zhao W, Shu Y, Gao M et al. (2017). Let-7a regulates expression of beta1-adrenoceptors and forms a negative feedback circuit with the beta1-adrenoceptor signaling pathway in chronic ischemic heart failure. Oncotarget 8: 8752-8764.

Fong CS, Mitchell P, Rochtchina E, Cugati S, Hong T, Wang JJ (2014). Three-year incidence and factors associated with posterior capsule opacification after cataract surgery: The 
Australian Prospective Cataract Surgery and Age-related Macular Degeneration Study. American Journal of Ophthalmology 157: 171-179 e171.

Hai B, Pan X, Du C, Mao T, Jia F et al. (2020). LncRNA XIST promotes growth of human chordoma cells by regulating miR-124-3p/ iASPP pathway. OncoTargets and Therapy 13: 4755-4765.

Hu C, Liu S, Han M, Wang Y, Xu C (2018). Knockdown of lncRNA XIST inhibits retinoblastoma progression by modulating the miR-124/STAT3 axis. Biomedicine \& Pharmacotherapy 107: 547-554.

Kim YS, Yi BR, Kim NH, Choi KC (2014). Role of the epithelialmesenchymal transition and its effects on embryonic stem cells. Experimental \& Molecular Medicine 46: e108.

Lee JS, Chung CC, Lin KK, Yu KH, Kuo CF, See LC (2016). Time trends in cataract surgery and after-cataract laser capsulotomy in Taiwan: A population-based retrospective cohort study. International Journal of Surgery 36: 265-273.

Li B, Mao R, Liu C, Zhang W, Tang Y, Guo Z (2018). LncRNA FAL1 promotes cell proliferation and migration by acting as a CeRNA of miR-1236 in hepatocellular carcinoma cells. Life Sciences 197: 122-129.

Li J, Xue W, Wang X, Huang W, Wang XX et al. (2019). HSP90 as a novel therapeutic target for posterior capsule opacification. Experimental Eye Research 189: 107821.

Li SL, Gao HL, Lv XK, Hei YR, Li PZ et al. (2017). MicroRNA-124 inhibits cell invasion and epithelial-mesenchymal transition by directly repressing Snail2 in gastric cancer. European Review for Medical and Pharmacological Sciences 21: 3389-3396.

Liang H, Yu T, Han Y, Jiang H, Wang C et al. (2018). LncRNA PTAR promotes EMT and invasion-metastasis in serous ovarian cancer by competitively binding miR-101-3p to regulate ZEB1 expression. Molecular Cancer 17: 119.

Liang YJ, Wang QY, Zhou CX, Yin QQ, He M et al. (2013). MiR-124 targets Slug to regulate epithelial-mesenchymal transition and metastasis of breast cancer. Carcinogenesis 34: 713-722.

Loda A, Heard E (2019). Xist RNA in action: Past, present, and future. PLoS Genetics 15: e1008333.

Nibourg LM, Gelens E, Kuijer R, Hooymans JM, Van Kooten TG, Koopmans SA (2015). Prevention of posterior capsular opacification. Experimental Eye Research 136: 100-115.

Puisieux A, Brabletz T, Caramel J (2014). Oncogenic roles of EMTinducing transcription factors. Nature Cell Biology 16: 488-494.

Sabanayagam C, Banu R, Chee ML, Lee R, Wang YX et al. (2019). Incidence and progression of diabetic retinopathy: a systematic review. The Lancet Diabetes \& Endocrinology 7: 140-149.

Shen J, Hong L, Yu D, Cao T, Zhou Z, He S (2019). LncRNA XIST promotes pancreatic cancer migration, invasion and EMT by sponging miR-429 to modulate ZEB1 expression. International Journal of Biochemistry \& Cell Biology 113: 17-26.

Wang X, Zhang G, Cheng Z, Dai L, Jia L et al. (2018). Knockdown of LncRNA-XIST suppresses proliferation and TGF- $\beta 1$-induced EMT in NSCLC through the Notch-1 pathway by regulation of miR-137. Genetic Testing and Molecular Biomarkers 22: 333-342.

Wang Y, Chen L, Gu Y, Wang Y, Yuan Y et al. (2019). LncRNA FEZF1-AS1 promotes TGF- $\beta 2$-mediated proliferation and migration in human lens epithelial cells SRA01/04. Journal of Ophthalmology 2019: 4736203.

Wang YN, Pei C, Qin L, Li JM, Yi JL, Chen L (2016). The expression of transcription factors Snail and Slug in epithelialmesenchymal transition of human lens epithelial cells induced by transforming growth factor-beta2. Zhonghua Yan Ke Za Zhi 52: 285-290.

Weatherbee BAT, Barton JR, Siddam AD, Anand D, Lachke SA (2019). Molecular characterization of the human lens epithelium-derived cell line SRA01/04. Experimental Eye Research 188: 107787.

Wei YL, Sun H (2019). Identification of hsa-mir-34a, hsa-mir-124, and hsa-mir-204 as signatures for cataract. Journal of Cellular Physiology 234: 10709-10717.

Wernecke L, Keckeis S, Reichhart N, Strauss O, Salchow DJ (2018). Epithelial-mesenchymal transdifferentiation in pediatric lens epithelial cells. Investigative Ophthalmology \& Visual Science 59: 5785-5794.

Westling AK, Calissendorff BM (1991). Factors influencing the formation of posterior capsular opacities after extracapsular cataract extraction with posterior chamber lens implant. Acta Ophthalmol (Copenh) 69: 315-320.

Wu C, Liu Z, Ma L, Pei C, Qin L et al. (2017a). MiRNAs regulate oxidative stress related genes via binding to the 3' UTR and TATA-box regions: A new hypothesis for cataract pathogenesis. BMC Ophthalmology 17: 142.

Wu CR, Ye M, Qin L, Yin Y, Pei C (2017b). Expression of lens-related microRNAs in transparent infant lenses and congenital cataract. International Journal of Ophthalmology 10: 361-365.

Xiao D, Cui X, Wang X (2019). Long noncoding RNA XIST increases the aggressiveness of laryngeal squamous cell carcinoma by regulating miR-124-3p/EZH2. Experimental Cell Research 381: $172-178$.

Xiong Y, Wang L, Li Y, Chen M, He W, Qi L (2017). The long noncoding RNA XIST interacted with MiR-124 to modulate bladder cancer growth, invasion and migration by targeting androgen receptor (AR). Cellular Physiology and Biochemistry 43: 405-418.

Zhang B, Chen Y, Qiu M, Ding Z (2017). Long noncoding RNA expression profile in HLE B-3 cells during TGF- $\beta 2$ induced epithelial-mesenchymal transition. BMC Ophthalmology 17: 69 .

Zhang RP, Xie ZG (2020). Research progress of drug prophylaxis for lens capsule opacification after cataract surgery. Journal of Ophthalmology 2020: 2181685.

Zhang Z, Zhu H, Liu Y, Quan F, Zhang X, Yu L (2018). LncRNA HOTAIR mediates TGF-beta2-induced cell growth and epithelial-mesenchymal transition in human lens epithelial cells. Acta Biochimica et Biophysica Sinica 50: 1028-1037.

Zhao Z, Zhou W, Han Y, Peng F, Wang R et al. (2017). EMT-Regulome: A database for EMT-related regulatory interactions, motifs and network. Cell Death \& Disease 8: e2872.

Zong Y, Zhang Y, Hou D, Xu J, Cui F et al. (2020). The lncRNA XIST promotes the progression of breast cancer by sponging miR125b-5p to modulate NLRC5. American Journal of Translational Research 12: 3501-3511. 